Journal of Mathematics and Informatics

Vol. 11, 2017, 147-153

ISSN: 2349-0632 (P), 2349-0640 (online)

Published 11 December 2017

www.researchmathsci.org

DOI: http://dx.doi.org/10.22457/jmi.v11a19

Journal of

Mathematics and

Informatics

\title{
Image Compression Using SPIHT Techniques
}

\section{K.Kalaiselvi}

\author{
Department of Computer Science, PVER College \\ Tiruchirappalli-23,Tamil Nadu, India \\ E-mail: kalaiselvig11984@gmail.com
}

Received 28 November 2017; accepted 7 December 2017

Abstract. A Digital Image Compression has attracted the interest of many Researchers. SPIHT based image Compression is a fast and efficient technique for Compression. In this Paper SPIHT Image Compression using Wavelet Transform (WT) is implemented with MatLab programming. The results are compressed in terms of PSNR, CR, and MSE paper concludes with the results of PSNR gives better compression.

Keywords: Wavelet Transform, SPIHT, PSNR, CR and MSE.

AMS Mathematics Subject Classification (2010): 65T60, 68U10, 94A08

\section{Introduction}

Digital image compression is now essential. Internet teleconferencing, High Definition Television (HDTV), satellite communications and digital storage of images will not be feasible without a high degree of compression. Wavelets became popular in past few years in mathematics and digital signal processing area because of their ability to effectively represent and analyse data. Typical application of wavelets in digital signal processing is Image compression. Image compression algorithms based on Discrete Wavelet Transform (DWT), such as statistical peak signal to noise ratio (PSNR) and Compression Ratio (CR) of the image. SPIHT is one of the best algorithms in terms of the peak signal-to-noise ratio (PSNR) and execution time. Set partitioning in hierarchical trees provide excellent rate distortion performance with low encoding complexity.

\section{Preliminaries}

Basvanthaswami et al. proposed an image compression and transmission algorithm which is suitable for low bit rate applications over Internet or any other wireless network [1]. Amritha et al. Wavelet Compression is a form of data compression well suited for image compression (also Video Compression and Audio Compression). The goal is to store image data in as little space as possible in a file. Wavelet compression can be either lossless or lossy. This means that the transient elements of a data signal can be represented by a smaller amount of information than would be the case if some other transform, such as the more widespread discrete cosine transform. The main advantage of wavelet transform over Discrete Cosine Transform (DCT) is that it has both time and frequency localization ability, which result in better performance in compression [2]. Sunitha et al. proposed SPIHT algorithm the analysis which was been performed on the 


\section{K.Kalaiselvi}

images and the performance measures are tabulated in the tables of different wavelets. Among all the wavelets $\mathrm{Bi}$ - Orthogonal wavelet performs well in both color and grey scale images [3]. Raj proposed SPIHT algorithm proves and promises that it can compress and reconstruct the image without changing the originality and the quality of the input image. From the experiment results it can be concluded that the SPIHT algorithm is effective and efficient than the existing approaches in terms of PSNR, MSE and computational time [4]. Vipin et al. proposed an implementing an image compression technique in FPGA. The combination of DWT and SPIHT algorithm is used for image compression. Set Partitioning in Hierarchical Trees (SPIHT) is a wavelet based image compression method that offers good image quality, fast coding, and high PSNR. It is used for lossless image compression [5]. Dodla et al proposed the SPIHT algorithm depends on Spatial Orientation Trees (SOT) defined on dyadic sub band structure, so some problems will arise because of its adaptation to WP decomposition. It must be resolved in order that SOT structure with well-defined parent-child relationships for arbitrary wavelet decomposition created Image compression using Wavelet and SPIHT Encoding Scheme [6]. Dnyaneshwar et al. proposed SPIHT algorithm using three different grayscale images with different compression techniques. For this performance analysis we have considered three parameters that is Compression Ratio, Peak Signal to Noise Ratio and Mean Square Error one by one are calculated [7]. A. Mallaiah et al proposed Retinex algorithm, images usually using all are analyzed, we use 5-level pyramids constructed with the 9/7 tap filters. Proposing a simple and effective method combined with Huffman encoding for further compression. In this paper a lot of bits are saves for the data transmission and storage purpose. In this decoding process uses the Retinex Algorithm for clear vision and quality improvement [8]. Sadashivappa et al. proposed SPIHT algorithm using Compressing color images efficiently are one of the main problems in multimedia applications. So we have tested the efficiency of color image compression using SPIHT algorithm. The SPIHT algorithm is applied for luminance $(\mathrm{Y})$ and chrominance $(\mathrm{Cb}, \mathrm{Cr})$ part of $\mathrm{RGB}$ to $\mathrm{YCbCr}$ transformed image. Reconstructed image is verified using human vision and PSNR. Huffman and arithmetic coding can be added to increase the compression [9]. William A. Pearlman proposed SPIHT is a simple and efficient algorithm with many unique and desirable properties. Efficient lossy reconstruction produced from truncation of file - scalable in fidelity [10].

\section{Proposed work}

The Set Partitioning in Hierarchical Tree algorithm (SPIHT) is proposed and utilized for lossless image compression nowadays. One of the most powerful wavelet based image compression techniques is SPIHT. The main advantages of SPIHT method are it can provide Good Image quality with high PSNR and it is the best method for progressive Image transmission. First, the image is decomposed into four sub-bands. The decomposition process is repeated until reach the final scale. Decomposition consists of one low-frequency sub-band with three high-frequency sub-bands.

\section{The compression consists of two parts:}

- DWT: Discrete Wavelet Transform

- SPIHT Encoder: encoder for information compaction. 
Image Compression Using SPIHT Techniques

The block diagram for the compression of images using SPIHT as

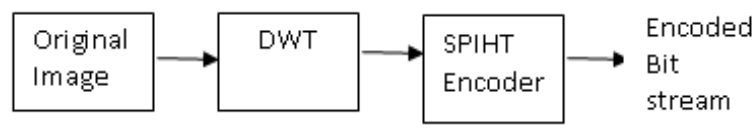

Figure 3.1: Block diagram: image compression

The DWT is iteratively applied to the image which results in a decomposed image. The SPIHT encoder is applied to this decomposed image.

The decompression consists again of two parts:

- SPIHT Decoder: decoder for information reconstruction

- IDWT: Inverse Discrete Wavelet Transform

The flow diagram of decompression image as

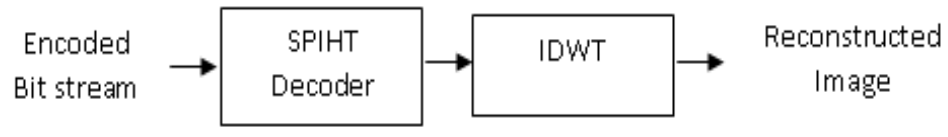

Figure 3.2: Block diagram: image decompression

1. SPIHT: A set partitioning in hierarchical trees algorithm and it will be eliminated from the subsets, then inserted into the LSP or it will be inserted into the LIP. In the refinement process, the $\mathrm{n}^{\text {th }}$ MSB of the coefficient in the LSP is taken as the final output. The value of $\mathrm{n}$ is decreased, again sorting with refinement is applied until $n=0$. Since, SPIHT algorithm controlled the bit rate exactly and the execution can be terminated at any time. Once the encoding process is over, then the decoding process is applied.

2. CR-Compression Ratio: The ratio of the size of the input Image to the size of the compressed bit stream. It is a measure performance of the compression algorithm.

\section{Compression Ratio = Output file size (bytes) / Input file size (Bytes)}

3. Mean Square Error (MSE): MSE is the metric used to verify the mean square error of the image. The MSE is used to estimate the difference between two images in terms squared error value.

$$
M S E=\mathrm{S}(\text { Sum }(\text { SquaredErrorImage })) /(\text { row } \times \text { columns })
$$

4. PSNR-Peak Signal Noise Ratio: PSNR is used to find out the ratio between the maximum power of a signal and the noise corrupted signal that affects the reliability of the signal representation. It is a measurement to measure the quality 


\section{K.Kalaiselvi}

of the image. The high PSNR value denotes the reconstructed image quality is high, and the low PSNR value denotes the reconstructed image quality is low.

$$
P S N R=10 \cdot \log _{10}\left((255)^{2} / M S E\right)
$$

\section{Experimental result}

Various bmp images size, quality, format are compressed using SPIHT Techniques. The quality of Images are Evaluated by the MSE, PSNR, and CR values are computed for each Image before and after Compression. Figure 4.1a displays the Input Image for Encoding process results with SPIHT algorithm, while Figure 4.b displays the Decoding process using SPIHT algorithm. Table4.1 shows the effectiveness of SPIHT algorithm such as MSE, PSNR, and CR.

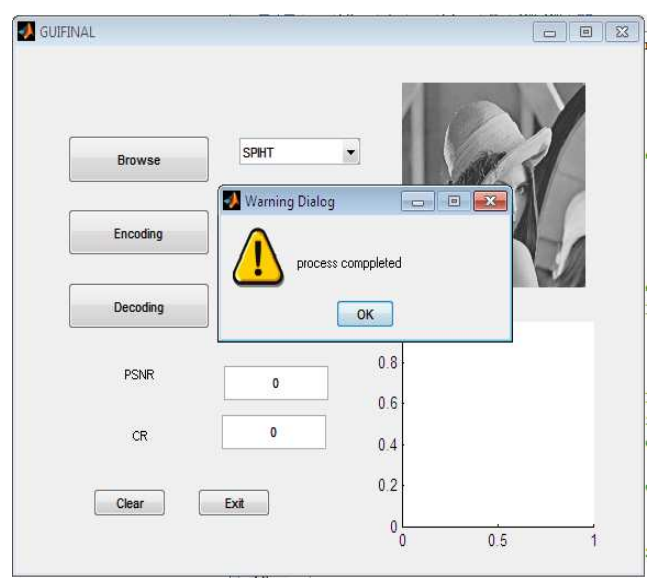

(a) SPIHT algorithm for Encoding

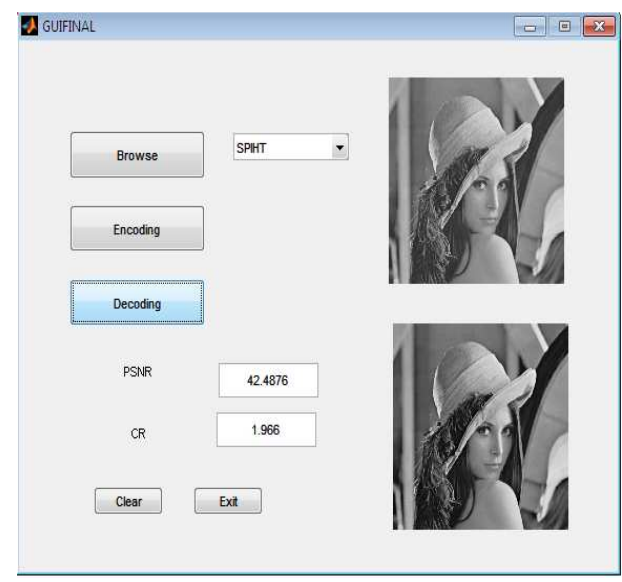

(b) SPIHT algorithm after Decoding

Table 4.1: Performance of Image Compression using SPIHT Technique

\begin{tabular}{|l|l|l|l|c|c|c|c|c|c|c|c|}
\hline \multicolumn{9}{|c|}{ Encoding Time } & \multicolumn{6}{c|}{ Decoding Time } & TR \\
\hline $\begin{array}{l}\text { Imag } \\
\text { e } \\
(\text { Bmp } \\
\text { ImW }\end{array}$ & $\begin{array}{l}\text { D } \\
\text { Time } \\
(\mathrm{sec})\end{array}$ & $\begin{array}{l}\text { cSPIH } \\
\text { T Time } \\
(\mathrm{sec})\end{array}$ & $\begin{array}{l}\text { PSN } \\
\text { R }\end{array}$ & CR & $\begin{array}{l}\text { Total } \\
\text { Encodin } \\
\text { g Time } \\
(\mathrm{sec})\end{array}$ & $\begin{array}{l}\text { dSPIH } \\
\text { T Time } \\
(\mathrm{sec})\end{array}$ & $\begin{array}{l}\text { IDW } \\
\text { T } \\
\text { Time } \\
(\mathrm{sec})\end{array}$ & $\begin{array}{l}\text { Total } \\
\text { Decodin } \\
\text { g Time } \\
(\mathrm{sec})\end{array}$ & $\begin{array}{l}\text { PSN } \\
\text { RdB }\end{array}$ & MSE & $\begin{array}{l}\text { CR } \\
\text { dB }\end{array}$ \\
\hline $\begin{array}{c}\text { lena_ } \\
\text { gray }\end{array}$ & 0.277 & 14.98 & 0 & 0 & 15.264 & 3.245 & 0.095 & 3.34 & 42.48 & 03.67 & 53 \\
\hline $\begin{array}{c}\text { barba } \\
\text { ra_gr } \\
\text { ay }\end{array}$ & 0.084 & 08.53 & 0 & 0 & 08.618 & 3.244 & 0.054 & 3.29 & 36.57 & 14.32 & 52 \\
\hline
\end{tabular}


Image Compression Using SPIHT Techniques

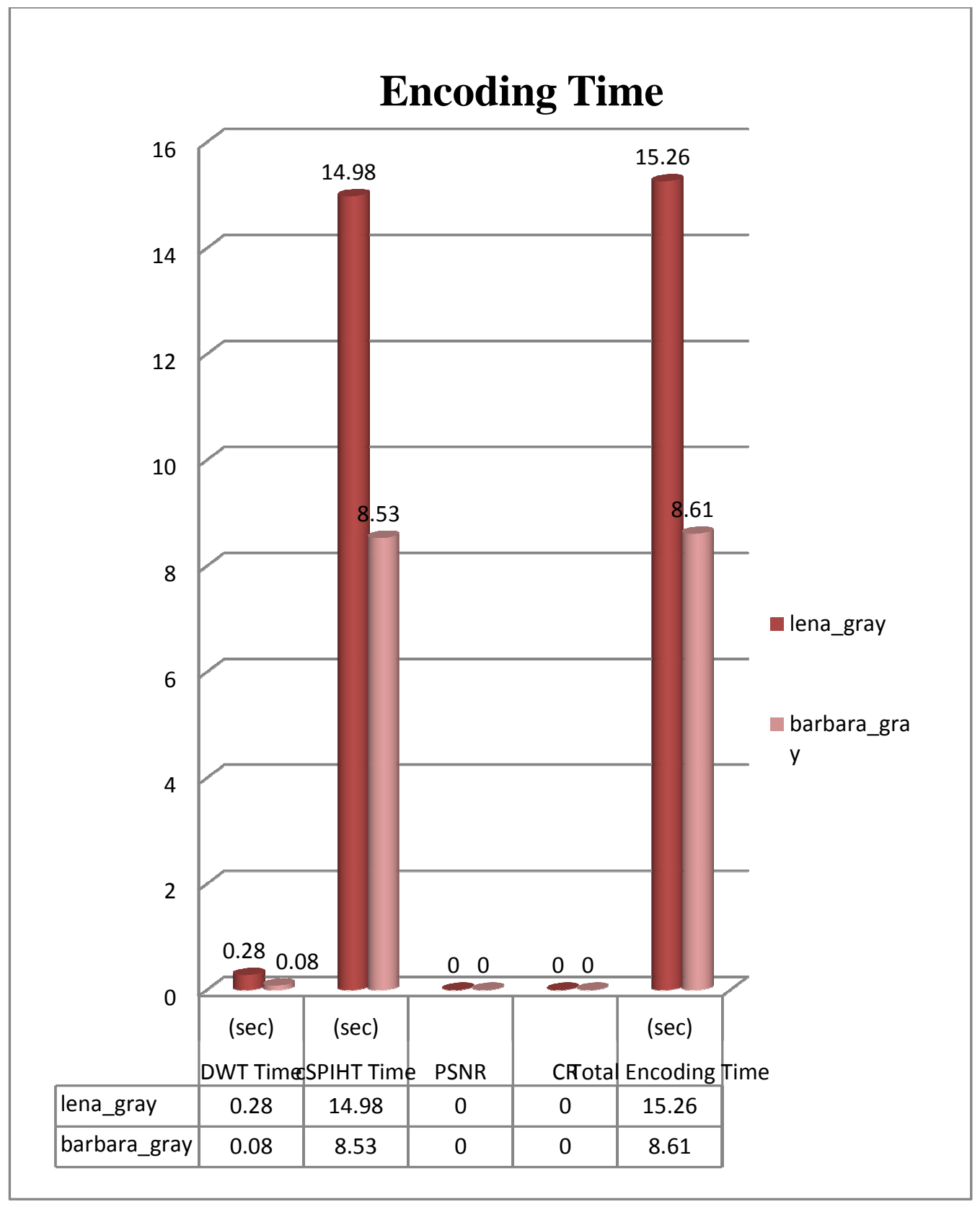

Figure 4.2: Comparison graph for encoding time

The Figure4.2 shows that the time taken to encode the images using SPIHT technique. The encoding time for lena_gray is 15.26 seconds and barbara_gray is 8.61 seconds. 
K.Kalaiselvi

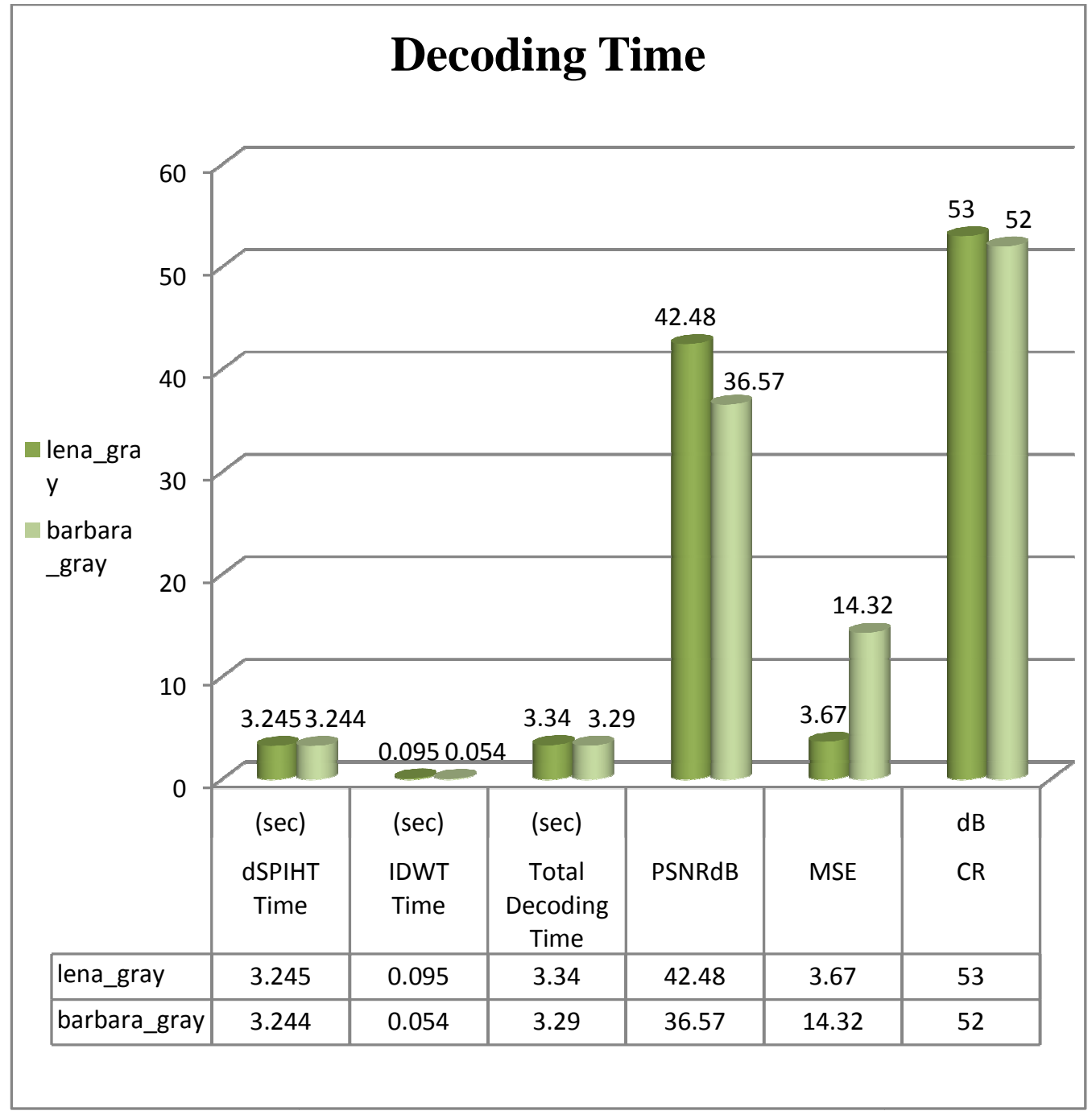

Figure 4.3: Comparison graph for decoding time

The pictorial representation of decoding time using SPIHT technique is shown on Figure4.3.The compression ratio, PSNR and MSE values of the image barbara_gray is 52 $\mathrm{dB}, 36.57 \mathrm{~dB}$ and 14.32 .

\section{Conclusion}

The proposed work introduced an Image Compression using SPIHT Technique based on DWT and IDWT methods. This technique achieves better results and concludes the result is effective and efficient in terms of MSE, PSNR, and CR is used to store less memory and better quality of compression. This technique will be acceptable by Medical community. Further enhancement may be produced by introducing any novel approaches 
Image Compression Using SPIHT Techniques

from different paradigms. In future this SPIHT algorithm will be modified to compress the 3-D colour images.

Acknowledgement. I would like to thank my family: my parents and to my brothers and sister for supporting me research throughout writing this thesis and my life in general.

\section{REFERENCES}

1. V.Basvanthaswami and T.Somasekar, Image compression using SPIHT, International Journal of Innovative Research in Computer and Communication Engineering, 5(2) (2017) 1840-1844.

2. K.M.Amritha and S.S.Nithin, Adaptive encoding and decoding of compressed video using SPIHT algorithm, International Journal of Advanced Research in Computer Engineering \& Technology, 4(5) (2015) 2409-2412.

3. P.Sunitha and J.L.Srinivas, Performance analysis of SPIHT algorithm in image compression, International Journal of Science, Engineering and Technology Research, 4(1) (2015) 1-166-170.

4. S.Nirmal Raj, SPIHT: A set partitioning in hierarchical trees algorithm for image compression, Contemporary Engineering Sciences, 8 (2015) 263-270.

5. V.Vipin and M.Mathews, FPGA implementation of image compression using SPIHT algorithm,International Journal of Advanced Research in Electrical, Electronics and Instrumentation Engineering, 3(1) (2014) 7001-7007.

6. S.Dodla, Y.D.SolmonRaju and K.V.Murali Mohan, image compression using wavelet and SPIHT encoding scheme, International Journal of Engineering Trends and Technology, 4(9) (2013) 3863-3865.

7. K.Dnyaneshwar and CH.Suneetha, SPIHT algorithm with Huffman encoding for image compression and quality improvement over MIMO OFDM channel, International Journal of Advanced Computer Research, 3(4) (2013) 651-700.

8. Mallaiah, S.K.Shabbir and T.Subhashini, An SPIHT algorithm with huffman encoder for image compression and quality improvement using Retinex slgorithm, International Journal of Scientific \& Technology Research, 1(5) (2012) 45-49.

9. Sadashivappa, K.V.S.AnandBabu and K.Srinivas, Color image compression using SPIHT algorithm, International Journal of Computer Applications, 16(7) (2011) 3442.

10. W.A.Pearlman, The set partitioning in hierarchical trees (SPIHT) algorithm, http://www.cipr.rpi.edu/research/SPIHT/EW_Code/SPIHT@JPEG2K_c97.pdf 